\title{
Staphylococcus aureus is a major pathogen in severe acute bacterial rhinosinusitis *
}

A. Byrjalsen, T. Ovesen, T. Kjaergaard

Department of Otorhinolaryngology, Head \& Neck Surgery, Aarhus University Hospitals, Aarhus, Denmark
Rhinology 52: 48-52, 2013

DOl:10.4193/Rhino13.004

*Received for publication:

January 17,2013

Accepted: August 16, 2013

\section{Abstract}

Purpose: The purpose of this study was to investigate the epidemiology and microbiology of severe acute bacterial rhinosinusitis in patients admitted to a Danish tertiary hospital.

Methods: A retrospective study was performed including all cases of acute rhinosinusitis admitted to the ENT-department of Aarhus University Hospital, in the period 2001 to 2011.

Results: In total, 143 cases of acute rhinosinusitis were identified of which 51\% were children. The most prevalent bacterial strains were Staphylococcus aureus followed by group A streptococcus, Haemophilus influenzae and Streptococcus pneumonia. Morexella catarrhalis was only rarely cultured. Anerobes were not assessed routinely. Of all patients, $47.8 \%$ presented with orbital complications and $2.1 \%$ developed intracranial complications. Patients infected with group A streptococcus had significantly higher leukocyte and neutrophil counts than other patients. All cultured S. aureus were resistant to penicillin, but sensitive to methicillin.

Conclusion: Our results imply that S. aureus and group A streptococcus are important pathogens in severe and complex cases of ABRS, in addition to the accepted pathogens S. pneumoniae and H. influenzae. On the contrary M. catarrhalis appears less significant. These findings have important implications regarding the selection of relevant treatment strategy in secondary care, which may currently be underestimating the role of $S$. aureus.

Key words: Staphylococcus aureus, streptococcus, sinusitis, microbiology, epidemiology

\section{Introduction}

Acute rhinosinusitis is a frequent health problem both in children and adults. It is most commonly caused by a viral upper respiratory tract infection, whereas bacterial involvement is less frequent, accounting for approximately $0.5-2 \%{ }^{(1)}$ in adults and $6-13 \%{ }^{(2)}$ in children. Most cases resolve spontaneously and severe complications are quite rare. At our institution, acute bacterial rhinosinusitis (ABRS) accounts only for a minority of admitted cases of acute infectious disease ${ }^{(3)}$.

S. pneumoniae, $H$. influenzae, and M. catarrhalis are generally considered the predominant bacterial species implicated in ABRS. Although recent literature indicates a different microbial profile in more severe cases of ABRS (4), the epidemiological and microbiological characteristics of this subgroup remains poorly described.

The objective of this study was to explore the epidemiology and bacteriology in patients with severe ABRS admitted to the ENT-department at a tertiary medical centre, Aarhus University Hospital, Denmark. Additionally, we wanted to study possible associations between clinical and paraclinical data and the major pathogens associated with ABRS.

\section{Materials and methods}

\section{Patient population}

The medical records of all patients with acute rhinosinusitis 
admitted to the ENT-department of Aarhus University Hospital, Denmark from January 2001 to December December 2011, were subjected to a review. Charts were retrieved by searching for relevant ICD-10 codes. The following variables were recorded: age, gender, duration of preadmission symptoms, type of antimicrobial therapy prior to admission and during hospitalization, type of surgical intervention and duration of hospitalization. C-reactive protein level (CRP), leukocyte count, and neutrophil count were registered. Additionally, paranasal sinus involvement as well as type and extend of orbital and intracranial complications were evaluated based on CT scans. Bacterial strains were recorded, and resistance patterns were registered. Specimens for bacteriological analyses were obtained primarily by swabs from the nasal cavity (middle meatus) or by aspirates from abscesses or infected sinuses. The samples were either sealed in the syringes (in the case of the aspirates) or placed in Stuarts transport medium and transported to the department of Clinical Microbiology. The samples were cultured on $5 \%$ blood agar plates with a band of staphylococcus across them (allowing for $\mathrm{H}$. influenzae to grow), incubated with $5 \% \mathrm{CO}_{2}$ at $35^{\circ} \mathrm{C}$ for 48 hours, and subsequently analysed for the growth of microorganisms.

\section{Statistical analysis}

Microbial profiles were analyzed across age groups and gender as well as infection related parameters. For statistical analysis Students $t$ test and analysis of variance (ANOVA) test was used when the data were normally distributed, whereas Kruskal-Wallis test was used for non-parametric data. Statistical significance was defined as $p<0.05$. Statistical analysis was preformed using STATA12.

\section{Results}

In total, 143 charts were reviewed. Females accounted for $36 \%$ of the sample and $51 \%$ were children ( $0-16$ years). The median duration of symptoms was 5.5 days, and the median duration of hospitalization was 4 days. Orbital complications were registered in $47.8 \%$ of cases, predominantly children (Table 1 ) and $2.1 \%$ developed intracranial complications. Isolated sinus puncture (maxillary sinus) was performed in $24.5 \%$ and sinus surgery, primarily ethmoidectomy and antrostomy was performed in $39.2 \%$ of patients.

Demographic and clinical details, stratified by microbial profile, are summarized in Table 2. The number of admissions peaked in February and March, with 20 and 22 admissions, respectively, and reached a monthly low in June with only five admissions.

Culture of samples was performed in 114 patients, and a positive growth was detected in 70 patients (61.4\%). The bacteria most frequently registered (culture rate per isolate) were $S$. aureus
Table 1. Sinus involvement and distribution of orbital and intracranial complications.

\begin{tabular}{|c|c|c|c|}
\hline & All & Adults & Children \\
\hline Single sinus & $11(9.0 \%)$ & $9(14.3 \%)$ & $2(3.4 \%)$ \\
\hline Polysinusitis & $30(24.6 \%)$ & $28(36.5 \%)$ & 7 (11.9\%) \\
\hline Pansinusitis & $14(11.5 \%)$ & $7(11.1 \%)$ & $7(11.9 \%)$ \\
\hline \multicolumn{4}{|l|}{ Orbital involvement } \\
\hline Preseptal cellulitis & $18(14.8 \%)$ & $10(15.9 \%)$ & $8(13.5 \%)$ \\
\hline Orbital cellulitis & $22(18.0 \%)$ & $7(11.1 \%)$ & $15(25.4 \%)$ \\
\hline Subperiosteal abscess & $20(16.4 \%)$ & $5(7.9 \%)$ & $15(25.4 \%)$ \\
\hline Orbital abscess & $6(4.9 \%)$ & $1(1.6 \%)$ & $5(8.5 \%)$ \\
\hline Intracranial abscess & $1(0.8 \%)$ & $1(1.6 \%)$ & 0 \\
\hline
\end{tabular}

Number of patients (stratified by age) with single-, poly-, or pansinusitis, as well as sinus orbital involvement.

(20\%), group A streptococcus (S. pyogenes) (15\%), H. influenzae (13.8\%), and S. pneumonia (8.8\%). Other streptococcus species accounted for $18.8 \%$, mainly represented by nonhaemolytic streptococcus, haemolytic streptococcus, group C streptococcus, and S. anginosus. Other microbial species accounted for $23.6 \%$. M. catarrhalis was only detected in $3.8 \%$ of isolates (Table 3). The distribution of bacteria differed somewhat between the adult and the paediatric populations, but in both groups S. aureus and Group A streptococcus were amongst the most prevalent strains.

All S. aureus were resistant to penicillin and two patients were additionally resistant to erythromycin. No $S$. aureus were resistant to methicillin.

Patients infected with group A streptococcus displayed higher leukocyte $(p=0.0093)$ and neutrophil $(p=0.04)$ counts than patients infected with other bacteria. Based on the four most prevalent bacterial strains, analyses in the paediatric population revealed the highest CRP and leukocyte count in children infected with group A streptococcus and the lowest values in children infected with S. pneumonia. In the adult population $S$. pneumonia was associated with the highest CRP and leukocyte count. However, despite several apparent associations between clinical, paraclinical and microbial data, these did not reach statistical significance. The site of culture sample (nasal cavity, sinus aspirate) did not influence type of bacterial isolate. 
Table 2. Demographic and paraclinical data stratified by bacterial strain.

\begin{tabular}{|c|c|c|c|c|c|c|c|c|}
\hline & All & SA & StrP & HIB & GAS & Other str. & Other spp. & No growth \\
\hline \multicolumn{9}{|l|}{ All } \\
\hline Age & $17(0-81)$ & $40(3-63)$ & $15(2-74)$ & $11.5(1-63)$ & $10(1-38)$ & $33(11-50)$ & $34(2-50)$ & $15.5(0-81)$ \\
\hline CRP & $530.9(55.9)$ & $594.8(152.6)$ & $412.9(262.8)$ & 302.4 (193.8) & $741.0(200.2)$ & 730.1 (276.2) & 209.4 (133.2) & $582.1(104.8)$ \\
\hline Leukocytes & $11.7(0.4)$ & $10.8(0.6)$ & $10.6(1.1)$ & $13.2(1.9)$ & $15.2(2.1)$ & $12.5(2.0)$ & $9.9(1.1)$ & $10.2(0.6)$ \\
\hline Neutrophils & $8.3(0.4)$ & $7.4(0.6)$ & $7.8(1.1)$ & $9.2(1.4)$ & $11.5(2.0)$ & $9.9(1.9)$ & $7.5(0.9)$ & $7.2(0.5)$ \\
\hline \multicolumn{9}{|l|}{ Adults } \\
\hline Age & $38(17-81)$ & $51.5(25-63)$ & $38(17-74)$ & $41(34-63)$ & $27(18-38)$ & $42(18-50)$ & $35(18-50)$ & $44(17-81)$ \\
\hline CRP & $516.8(82.8)$ & $696.0(214.3)$ & 780.7 (583.4) & $149.5(106.5)$ & $702.7(243.3)$ & $945.0(412.4)$ & $39.2(15.7)$ & $429.5(16.3)$ \\
\hline Leukocytes & $10.4(0.5)$ & $10.6(0.8)$ & $11.0(1.9)$ & $11.2(2.5)$ & $8.1(0.4)$ & $12.5(3.2)$ & $9.4(0.9)$ & $8.9(0.6)$ \\
\hline Neutrophils & $7.5(0.5)$ & $\begin{array}{ll}7.8 & (0.7)\end{array}$ & $8.3(1.8)$ & $7.8(1.2)$ & $5.1(0.6)$ & 10.1 (3.1) & $7.0(0.9)$ & $5.9(0.5)$ \\
\hline \multicolumn{9}{|l|}{ Children } \\
\hline Age & $9.5(0-16)$ & $11.5(3-14)$ & $8.5(2-15)$ & $6(1-15)$ & $4(1-10)$ & $11(11-14)$ & $11(2-14)$ & $11(0-16)$ \\
\hline CRP & $546.3(75.2)$ & $426.2(198.2)$ & $137.2(111.3)$ & 363.6 (272.8) & 768.3 (312.1) & 353.9 (172.9) & $635.0(341.0)$ & 720.7 (146.2) \\
\hline Leukocytes & $13.1(0.6)$ & $11.2(1.1)$ & $10.4(1.5)$ & $13.8(2.5)$ & $20.3(1.8)$ & $12.4(0.9)$ & $10.9(3.8)$ & $11,3(0.9)$ \\
\hline Neutrophils & $9.2(0.6)$ & $6.5(1.1)$ & $7.5(1.6)$ & $9.6(1.8)$ & $16.1(1.9)$ & $9.5(1.0)$ & $8,6(2.8)$ & $8,3(0.8)$ \\
\hline
\end{tabular}

Age: median (range). CRP, leukocytes, and neutrophils: mean (standard error of mean). S. aureus (SA), S. pneumoniae (StrP), H. influenzae (HIB), group A streptococcus (GAS), other streptococcus species (other Str), other species (other spp).

Tabel 3. Culture rates.

\begin{tabular}{|c|c|c|c|}
\hline & All & Children & Adults \\
\hline No. of cultures & 114 & 57 & 57 \\
\hline No. of isolates & 80 & 36 & 44 \\
\hline No. of positive cultures & 70 & 32 & 38 \\
\hline \multicolumn{4}{|l|}{$\begin{array}{l}\text { Culture rate (per person/ } \\
\text { isolate), } \%\end{array}$} \\
\hline S. aureus & $22.9 / 20.0$ & $18.8 / 16.7$ & $26.3 / 22.7$ \\
\hline Group A streptococcus & $17.1 / 15.0$ & 21.9/19.4 & $13.2 / 11.4$ \\
\hline H. influenzae & $15.7 / 13.8$ & $18.8 / 16.7$ & $13.2 / 11.4$ \\
\hline S. pneumoniae & $10.0 / 8.8$ & $12.5 / 11.1$ & $7.9 / 6.8$ \\
\hline Morexella catarrhalis & $4.3 / 3.8$ & $3.1 / 2.8$ & $5.3 / 4.5$ \\
\hline Other streptococcus species & $21.4 / 18.8$ & $15.6 / 13.9$ & $26.3 / 22.7$ \\
\hline Other species & $22.9 / 19.8$ & $25.0 / 22.2$ & $21.0 / 18.2$ \\
\hline
\end{tabular}

Culture rates per isolate and per person in the entire sample, and stratified by age. "Other streptococcus" is mainly represented by nonhaemolytic streptococcus and to a lesser extend group C streptococcus, haemolytic streptococcus, and S. anginosus. "Other species" represent Pseudomonas, Proteus mirabilis, enterobactericae, Corynebacterium, and Fusobacterium necrophorum.
Prior to admission $47.5 \%$ of the patients received antibiotic therapy, most commonly penicillin (67.2\%). During hospitalization penicillin, administered intravenously, remained the drug of choice, followed by Cefuroxime. Of those receiving preadmission antibiotics, $39.7 \%$ had negative cultures, whereas of those not receiving preadmission antibiotics, $28.1 \%$ were negative. S. aureus was found in $15.5 \%$ of patients having received preadmission antibiotics and $10.9 \%$ of patients not treated with antibiotics prior to admission.

\section{Discussion}

We have demonstrated that S. aureus and group A streptococcus are frequently associated with severe cases of ABRS, in addition to those traditionally considered as major pathogens in ABRS (S. pneumoniae, $H$. influenzae) ${ }^{(5-7)}$. On the contrary, M. catarrhalis, characterized by a relatively low virulence ${ }^{(8)}$, seems to play a limited role. The potential relevance of $S$. aureus as a major pathogen in ABRS has been considered in previous studies. In a recent meta-analysis, the mean culture rates in adults were $32.7 \%$ for S. pneumonia, $31.7 \%$ for $H$. influenzae, $8.8 \%$ for $M$. catarrhalis, and $10.1 \%$ for S. aureus ${ }^{(4)}$. Based on these findings, the authors warranted a reconsideration of $S$. aureus as a major pathogen in ABRS. Subsequent studies on both adult and paediatric populations, have confirmed these findings ${ }^{(9-11)}$. Although group $A$ streptococcus is not considered a major pathogen in ABRS, it appears relatively common, and has by some been associated with ethmoiditis and orbital complications to ABRS. According to Conrad et al., group A streptococcus species are primarily seen causing ABRS in children ${ }^{(12)}$. This view is supported in our study 
with an apparent overrepresentation of ethmoiditis/orbital complications in patients infected with group A streptococcus, and a majority of children infected with group A streptococcus (average age 14.9 years) ${ }^{(13,14)}$.

The microbial profile of our sample, with a high culture rate of S. aureus and group A streptococcus and a low culture rate of M. catarrhalis, is the likely result of a selection of virulent and resilient bacterial strains, associated with severe disease i.e. purulent sinusitis and orbital complications. In addition, community-based first line antibiotic treatment of ABRS, mainly penicillin (15), may be associated with the apparent microbial overweight of S. aureus. This is reflected in our sample as well as in previous reports from our institution, where preadmission antibiotics has been associated with increased culture rates of S. aureus ${ }^{(16)}$. Although we found no association between culture site and technique and bacterial strain, we cannot completely rule out possible contamination i.e. the nasal vestibule and rhinopharynx in which case an overrepresentation of $S$. aureus would be likely. However, previous studies have also found consistency with regard to different culture sites and bacterial strains ${ }^{(17-19)}$. Although considered during case reviews, incomplete differentiation between subgroups of $A B R S$, e.g. odontogenic $A B R S$ and acute exacerbation of CRS, may constitute a confounder with possible effect on the microbial profile in selected cases $(5,9,20)$. The antibiotic sensitivities of the cultured organisms revealed that MRSA is currently not at problem in this Danish subpopulation as no such microbes were identified. Although the incidence of MRSA infections has increased in Denmark in recent years, it remains low compared to other European countries (21). Our updated report, which mav be considered representative for the country as a whole, indicates a continued low MRSA load in Denmark. This is the likely gain of a strict antibiotic policy. On the contrary, all S.aureus isolates were resistance to penicillin, likely due to $\beta$-lactamase activity. Thus, distribution of this enzyme appears widespread, and although microbial selection may influence resistance patterns in our population (discussed above), the findings are not surprising. Apart from two cases of erythromycin resistency, microbial susceptibilty patterns were as expected. However, our samples were not cultured under anaerobic conditions, thus we cannot say anything about this spectrum of microorganisms, as well as the possible influence on growth of co-cultures. However, anaerobes are generally not considered significant influential in ABRS.

According to our findings, $\beta$-lactamase stable antibiotics should be considered first choice in ABRS cases in secondary care, e.g. 2nd generation cephalosporines which are also active against Gram-negative bacteria. However, the current first line antimicrobial advise in primary care should remain unchanged, to avoid unnecessary evolution of microbial resistance.

Stratification based on microbial data revealed higher leukocyte and neutrophil counts in individuals, mainly paediatric patients, infected with group A streptococcus. In contrast, S. aureus and other major pathogens in our sample did not appear to be associated with a particularly poorer outcome. Thus, our results did not support findings in recent studies indicating a difference in severity between ABRS caused by S. pneumoniae and $H$. influenzae, the former resulting in a more severe progression of the disease ${ }^{(22)}$. However, a relatively low-powered analysis as well as a large variability within the outcome variables impaired the strength of our analyses.

A significant male preponderance was found in our sample. Similar findings have been presented previously ${ }^{(9,23-25)}$, but the reason for this is unclear.

Issues that might compromise comparability with other studies include differences in standards for assessment of bacterial cultures (i.e., cut-off values for colony count criteria), preadmission antibiotic treatment regimes, demographic composition, institutional standards for admission and treatment, and diagnostic criteria.

Our results imply that $S$. aureus and group A streptococcus are important pathogens in severe ABRS and acute complications to $A B R S$, in addition to the accepted pathogens S. pneumoniae and H. influenzae. On the contrary, M. catarrhalis appears less significant. These findings have important implications regarding the selection of relevant treatment strategy in secondary care, which may currently be underestimating the role of $S$. aureus.

\section{Acknowledgement}

Thank you to the Department of Clinical Microbiology, Aarhus University Hospital, Denmark for their help in finding results of microbiologic tests/culturing.

\section{Authorship contribution}

AB: Data collection and paper drafting. TO: Revision of paper. TK: Statistical analysis and revision of paper.

\section{Conflict of interest}

All authors: no conflicts.

\section{References}

1. Fokkens WJ, Lund VJ, Mullol J, et al. EPOS 2012: European position paper on rhinosinusitis and nasal polyps 2012. A summary for otorhinolaryngologists. Rhinology 2012; 50: 1-12.

2. Wald ER, Guerra N, Byers C. Upper
Respiratory Tract Infections in Young Children: Duration of and Frequency of Complications. Pediatrics 1991; 87: 129-133.

3. Brook I. Microbiology and antimicrobial treatment of orbital and intracranial complications of sinusitis in children and their management. Int J Pediatr
Otorhinolaryngol 2009; 73: 1183-1186.

4. Payne SC, Benninger MS. Staphylococcus aureus is a major pathogen in acute bacterial rhinosinusitis: a meta-analysis. Clin Infect Dis 2007; 45: 121-127.

5. Brook I. Microbiology and antimicrobial management of sinusitis. J Laryngol Otol 
2005: 119: 251-258.

6. Brook I. Microbiology of recurrent acute rhinosinusitis. Laryngoscope 2004; 114: 129131.

7. Wald ER. Acute otitis media and acute bacterial sinusitis. Clin Infect Dis 2011; 52(suppl 4): 277-283.

8. Anon JB, Jacobs MR, Poole MD, et al. Antimicrobial treatment guidelines for acute bacterial rhinosinusitis. Otolaryngol Head Neck Surg 2004; 130 (suppl 1): 1-45.

9. Liao S, Durand ML, Cunningham MJ. Sinogenic orbital and subperiosteal abscesses: microbiology and methicillinresistant Staphylococcus aureus incidence. Otolaryngol Head Neck Surg 2010; 143 392-396.

10. McKinley MS, Yen MT, Miller AM, Yen KG. Microbiology of paediatric orbital cellulitis. Am J Ophthalmol 2007; 144: 497-501.

11. Oxford LE, McClay J. Medical and surgical management of subperiosteal abscess secondary to acute sinusitis in children. Int J Pediatr Otorhinolaryngol 2006; 70: 18531861.

12. Conrad DA, Jenson HB. Management of acute bacterial rhinosinusitis. Curr Opin Pediatr 2002; 14: 86-90.

13. Brook I. Microbiology of sinusitis. Proc Am Thorac Soc 2011; 8: 90-100.

14. Fellan RL, Vallhonrat PR, Youssef FW, Aristazabal RJ, Cubells LC, Fernandez PJ. [Orbital and periorbital cellulitis. Review of 107 cases]. An Esp Pediatr 2000; 53: 567-572.

15. Lindbaek M, Melby KK, Schoyen R, Hjortdahl P. Bacteriological findings in nasopharynx specimens from patients with a clinical diagnosis of acute sinusitis. Scand J Prim Health Care 2001; 19: 126-130.

16. Rusan M, Klug TE, Ovesen T. An overview of the microbiology of acute ear, nose and throat infections requiring hospitalization. Eur J Clin Microbiol Infect Dis 2009; 28: 243251.

17. Benninger MS, Appelbaum PC, Denneny JC, Osguthorpe DJ, Stankiewicz JA. Maxillary sinus puncture and culture in the diagnosis of acute rhinosinusitis: the case for pursuing alternative culture methods. Otolaryngol Head Neck Surg 2002; 127: 7-12.

18. Casiano RR, Cohn S, Villasuso E 3rd, et al. Comparison of antral tap with endoscopically directed nasal culture. Laryngoscope 2001; 111: 1333-1337.

19. Talbot GH, Kennedy DW, Scheld WM, Granito K. Rigid nasal endoscopy versus sinus puncture and aspiration for microbiologic documentation of acute bacterial maxillary sinusitis. Clin Infect Dis 2001; 33: 1668-1675.

20. Brook I. Microbiology of acute sinusitis of odontogenic origin presenting with periorbital cellulitis in children. Ann Otol Rhinol Laryngol 2007; 116: 386-388.

21. http://www.sst.dk/Sundhed og forebyggelse/Smitsomme sygdomme/MRSA. aspx

22. Benninger M, Brook I, Farrell DJ. Disease severity in acute rhinosinusitis is greater in patients infected with streptococcus pneumoniae than in those infected with Haemophilus influenzae. Otolaryngol Head
Neck Surg 2006; 135: 523-528.

23. Sultezs M, Csakanyi Z, Majoros T, Farkas Z, Katona G. Acute bacterial rhinosinusitis and its complications in our pediatric otolaryngological department between 1997 and 2006. Int J Pediatr Otorhinolaryngol 2009; 73: 1507-1512.

24. Soler ZM, Sauer DA, Mace J, Smith TL. Relationship between clinical measures and histopathologic findings in chronic rhinosinusitis. Otolaryngol Head Neck Surg 2009; 141: 454-461.

25. Oxford LE, McClay J. Complications of acute sinusitis in children. Otolaryngol Head Neck Surg 2005; 133: 32-37.

A. Byrjalsen

Department of Otorhinolaryngology,

Head \& Neck Surgery

Aarhus University Hospitals

Noerrebrogade 44

8000 Aarhus C

Denmark

Tel: +45-602-49 580

E-mail: a.byrjalsen@hotmail.com 